\title{
In memoriam Peter S. Harper
}

\author{
Mark Rogers, Julian Sampson, Angus Clarke
}

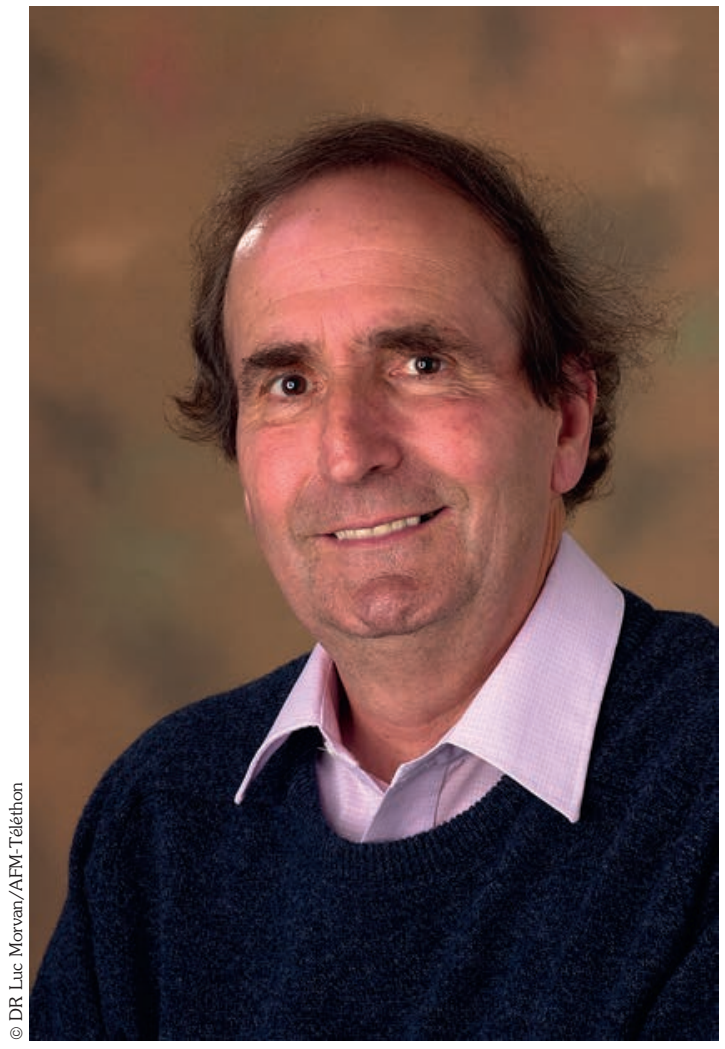

Peter Stanley Harper, l'un des généticiens cliniciens les plus remarquables et les plus respectés au monde, est né le 28 avril 1939 à Barnstaple dans le sud-ouest de l'Angleterre et est décédé le 23 janvier 2021 à l'âge de 81 ans. Son père, le Dr Richard Harper, était médecin généraliste dans cette même ville, et avait développé un vif intérêt académique pour les liens entre évolution et maladies. La mère de Peter, Margery (née Elkington), était une talentueuse spécialiste de la langue française, diplômée d'Oxford puis de la Sorbonne où elle avait obtenu un doctorat. Elle avait alors sacrifié une carrière universitaire prometteuse pour suivre le travail de son mari. Peter a grandi avec un amour profond de la nature et de la campagne environnante du Devon. Après avoir envisagé un temps une carrière directement en rapport avec ces centres d'intérêt, il décida plutôt de suivre les traces de son père en s'engageant dans des études de médecine. À l'Université d'Oxford (au prestigieux Exeter College), il suit, à partir de 1957, une formation complémentaire en génétique et en biologie dans le département de zoologie. En complément de ses études médicales et, après avoir terminé sa formation clinique à l'hôpital St Thomas de Londres en 1964, il décide, pour la suite de sa carrière, d'allier génétique et médecine. Peter n'avait pas de plan de carrière pré-établi pour y parvenir, et c'est pourquoi il a d'abord occupé des postes en pédiatrie et en médecine interne avant de déménager en 1967 à Liverpool où il a rapidement et naturellement trouvé sa place dans le groupe de Cyril Clarke (devenu plus tard Sir Cyril, président du Collège Royal des Médecins). Tout comme Peter, Cyril manifestait un intérêt majeur pour la génétique. Un engouement qui s'était développé grâce à ses recherches sur le papillon à queue d'hirondelle. Cyril avait récemment créé l'unité de génétique médicale Nuffield à la faculté de médecine. Au cours de ses deux années passées à Liverpool, Peter avait poursuivi sa formation médicale et travaillé sur les formes héréditaires de cancer de l'œesophage à la faculté de médecine tout en s'intéressant à la génétique des insectes dans le département de zoologie.

Peter et Elaine, qui s'étaient mariés à Liverpool en 1968, ont ensuite déménagé en 1969 à Baltimore aux USA où Peter bénéficia d'une bourse de recherche de deux ans chez Victor McKusick à la Johns Hopkins School of Medicine. Il s'est alors particulièrement intéressé à la neurogénétique à cette occasion et a pu conduire une étude spécifique sur la dystrophie myotonique de type 1 (maladie de Steinert). Ce travail a permis d'établir un lien entre la maladie et des marqueurs de groupe sanguin appelés Lu et $\mathrm{ABH}$. Ces marqueurs biologiques et la dystrophie myotonique avaient tendance à cosegréger dans les familles, laissant supposer que ces loci étaient proches les uns des autres sur le même chromosome. Ceci constitua une première étape importante vers l'identification du gène causal et conduisit à une série de publications et à une thèse que Peter soutiendra finalement en 1972 à Oxford.

De retour au Royaume-Uni en 1971, Peter choisit de prendre l'équivalent d'un poste de maitre de conférences à la faculté de médecine de Cardiff, au sein de l'Université du Pays de Galles. À partir du service de médecine où son poste se trouvait, il a alors tissé des liens étroits avec des biochimistes, des 
anatomopathologistes et d'autres professionnels qu'il a commencé à réunir pour créer officiellement un département de génétique médicale. Celui-ci s'est rapidement développé tant dans le domaine de la recherche que dans celui des activités cliniques dépendant du National Health Service (NHS). Au début, Peter s'est occupé du service clinique seul, tout en prenant part au travail hospitalier traditionnel consacré à la pathologie aiguë. L'équipe clinique s'est rapidement étoffée ce qui a permis de mieux répartir le travail de prestation de services dispensé à travers tout le Pays de Galles. Les programmes de recherche de Peter se sont ensuite concentrés particulièrement sur les dystrophies musculaires et la maladie de Huntington (MH), Cardiff devenant rapidement un centre de renommée internationale pour ces deux pathologies.

En 1987, après des années de préparation, le projet de Peter d'un centre intégrant université et NHS s'est concrétisé par l'ouverture, sur le campus de l'hôpital universitaire du Pays de Galles, de l'Institut de génétique médicale. Un bâtiment relativement petit accueillait des consultations dispensées par des cliniciens et des conseillers en génétique, des équipes de génétique moléculaire du NHS et de l'université, des cytogénéticiens, un laboratoire de dépistage biochimique néonatal, des activités de pathologie fotale, des experts en bio-informatique, des spécialistes des sciences sociales, des psychiatres et des psychologues. La diversité de ces professionnels a créé un environnement unique dans lequel de nombreuses compétences et projets ont été mis à profit concernant les maladies héréditaires. Le projet à plus long terme d'identifier les gènes de la dystrophie myotonique de type 1 (DM1) et de la MH porté par Peter a été couronné de succès, grâce à une collaboration internationale, avec la découverte du gène responsable de la DM1 en 1992 et celui de la $\mathrm{MH}$ en 1993. Ces deux gènes étant le siège d'expansions répétées de triplets, un mécanisme pathologique totalement nouveau à l'époque. Des approches fondées sur les preuves ont été utilisées en matière de tests génétiques prédictifs. Des thématiques plus complexes telles que génétique et assurance, ou la pratique des tests génétiques chez les enfants ont été explorées dans le détail, ce qui a conduit à des recommandations qui ont été reprises à l'échelle internationale. Tout au long de ces entreprises, les points de vue des patients et de leurs familles ont toujours été pris en compte, et leurs organisations représentatives, comme le Myotonic Dystrophy Support Group et la Huntington's Disease Association, ont été impliquées en tant que partenaires à part entière. Un flot constant de scientifiques, de cliniciens et de conseillers en génétique se sont succédé pour se former à l'Institut. Beaucoup d'entre eux ont également été accueillis dans la maison de Peter et de sa femme Elaine pour des événements à caractère social, devenant ainsi des amis et des collègues de longue date. Nombre d'entre eux sont ensuite devenus des leaders dans leur domaine, travaillant dans d'autres centres et diffusant l'approche holistique de Peter à l'échelle nationale et internationale.

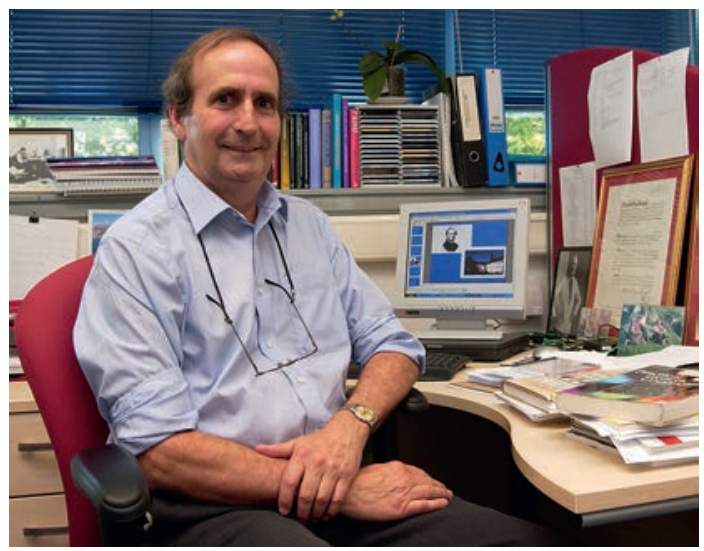

Tout au long de sa direction de l'Institut, le style de leadership de Peter est resté informel, généreux et bienveillant. Sa vieille camionnette VW décorée d'autocollants pacifistes fournissait un moyen de transport plutôt atypique pour un professeur de faculté, et ses pulls sans manches rayés n'étaient pas particulièrement à la mode. Sa sagesse, sa vision et sa capacité à anticiper les évolutions qui étaient en gestation, étaient universellement reconnues. Peter était également parfaitement capable de prendre des décisions difficiles et le fait est qu'il a presque invariablement fait le bon choix. Dans les rares occasions où les choses ne se sont pas déroulées comme il s'y attendait, il a transformé ce que d'autres auraient pu considérer comme des erreurs, ou des échecs à rapidement oublier, en occasions d'améliorer la façon dont les choses seraient faites à l'avenir, souvent en les publiant sous forme d'études de cas.

Comme pour tous ses écrits, les publications de Peter étaient claires et faciles d'accès. Peter est l'auteur de plus de 15 livres, certains destinés aux professionnels, d'autres aux patients. Il a même écrit une pièce de théâtre. Ses monographies sur la maladie de Steinert et la maladie de Huntington sont devenues des textes de référence pour les deux affections. Toujours désireux d'utiliser ses connaissances au bénéfice des malades, il a écrit le très populaire Myotonic Dystrophy: The Facts, un ouvrage publié depuis dans de nombreuses langues. Sa traduction et diffusion aux familles françaises ont 
été permises par le soutien de l'AFM-Téléthon. Cette dernière l'avait d'ailleurs invité à plusieurs reprises à des colloques scientifiques en tant que spécialiste incontesté de la maladie de Steinert. En génétique, son guide pratique de Conseil Génétique est aussi devenu un livre de référence, présent sur le bureau de nombreux professionnels. Traduit dans de nombreuses langues, il a fait l'objet de huit mises à jour dont la dernière par notre collègue Angus Clarke.

Peter a quitté son poste de chef de département en 2000. Avant de prendre sa retraite définitive en 2004, il avait commencé à développer un projet à long terme destiné à retracer l'histoire de la génétique médicale (Genetics and Medicine Historical Network). Ce travail se faisait principalement au travers d'entretiens avec ceux qui avaient été impliqués dans cette aventure, même si beaucoup d'entre eux étaient bien avancés en âge, et d'une analyse minutieuse de leurs archives. Ce travail est devenu une entreprise majeure, occupant Peter jusqu'à la fin de sa vie et impliquant de nombreux déplacements à l'étranger. Le projet comportait également une documentation minutieuse sur les mésusages passés et contemporains de la génétique en Europe, en Amérique, en Russie et en Chine. Ce travail important, en particulier l'enregistrement des témoignages personnels et des réflexions de cliniciens et de scientifiques, nécessitait un degré de confiance et de compréhension que seule une personne de la stature de Peter pouvait avoir. Une grande partie du matériel accumulé peut être lu dans ses livres, y compris "A Short History of Medical Genetics" (2008) et "Evolution of Medical Genetics - A
British Perspective" (2020) et consulté en ligne à www.genmedhist.org.

Au-delà de Cardiff, Peter Harper aura été très actif au sein de sociétés et de réseaux professionnels à l'échelle nationale et internationale, tels que la $\mathrm{Cli}$ nical Genetics Society, la British Society for Human Genetics (maintenant la BSGM) et le Royal College of Physicians; également au sein de l'European Society of Human Genetics, qu'il a aidé à réformer dans les années 1980, de l'American College of Medical Genetics, qui lui a décerné son prix pour l'ensemble de ses travaux, et de l'American Society of Human Genetics, dont il a été membre pendant plus de 50 ans. Il a été rédacteur en chef du Journal of Medical Genetics pendant 10 ans (1986-1996) et membre de la Human Genetics Commission et du Nuffield Council for Bioethics. Il a reçu le titre de commandeur de l'Empire Britannique (1994) et un titre de chevalier (2004) pour services rendus à la médecine et à la génétique médicale (mais il n'a jamais utilisé le titre).

Peter a toujours donné la priorité au temps passé en famille en tant que mari, père et grand-père. Il aimait particulièrement partager sa passion et sa connaissance approfondie de la nature et de la faune avec sa famille et rendait visite à son fils aîné et à sa famille en Australie chaque année. Peter laisse dans le deuil son épouse Elaine et leurs enfants, Mathew, Emma, Nicholas, Katy et Lucy.

In memoriam Peter S. Harper

Traduction libre de J. Andoni Urtizberea

LIENS D'INTÉRÊT

Les auteurs déclarent n'avoir aucun lien d'intérêt concernant les données publiées dans cet article.

\section{Retrouvez toutes les Actualités de la Myologie sur les sites de :}

la Société Française de Myologie www.sfmyologie.org

\author{
la filière de santé neuromusculaire FILNEMUS \\ www.filnemus.fr
}
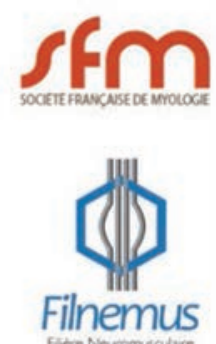